M. Roček

D.A.M.T.P., University of Cambridge, U.K.

and

$P \cdot K$. Townsend

CERN -- Geneva

\title{
ABSTRACT
}

We formulate the $N=4$ supersymmetric non-linear $\sigma$ model (in two spacetime dimensions) in terms of $\mathrm{N}=1$. four-dimensional superfields, and give the explicit form of all supersymetry transformations. Using supergraph techniques we show that all divergent contributions to the two-point function vanish up to the three loop level. We also show finiteness to leading order in the $1 / \mathrm{n}$ expansion. 


\section{INTRODUCTION AND RESULTS}

Non-linear $\sigma$ models are the field theorist's favorite two-dimensional playground for the investigation of properties conjectured to hold in more physical, and more complicated, four-dimensional theories. Supersymetric theories are known to have improved ultra-violet behaviour over their non-supersymmetric counterparts, in some cases rendering the theory finite, at least for the leading orders of perturbation theory ${ }^{1), 2)}$. The supersymetric non-linear $\sigma$ models ${ }^{3)}$ are therefore natural two-dimensional candidates to study in this regard, and have the advantage of being considerably simpler than four-dimensional theories. Freedman and Alvarez-Gaumé have recently studied the ultra-violet behaviour of general supersymmetric $\sigma$ models (where the Bose fields are the coordinates of an arbitrary Riemmannian manifold) with striking results ${ }^{4)}$. In particular, those models defined on Ricci flat manifolds are finite to two loops, as a consequence of general arguments and as verified by explicit calculation. Perhaps the most interesting supersymmetric $\sigma$ models are those with an extended $N=4$ supersymmetry $(\mathrm{N}=2$ in four dimensions); like the $\mathrm{N}=4$ super-Yang-Mills and the $\mathrm{N}=8$ supergravity such theories are self-conjugate. Remarkably, the only known example 5 ) of such an $\mathrm{N}=4$ supersymmetric $\sigma$ model is defined on a manifold with vanishing Ricci tensor ${ }^{6)}$, and therefore is one and two loop finite. It is important to investigate whether finiteness persists in higher orders, and from elegant general considerations it has been argued ${ }^{4)}$ that this is the case. We start our investigation of this problem by formulating the model in terms of $\mathrm{N}=1$ four-dimensional superfields, which by dimensional reduction are equivalent to $\mathrm{N}=2$ two-dimensional superfields. (Unless otherwise stated, our subsequent discussion is four-dimensional. In particular, the four two-dimensional supersymmetries will be referred to as two four-dimensional supersymmetries.) Because our formulation is in superspace one supersymmetry is manifest. The other one we give explicitly; it appears in a supermultiplet of transformations that includes the non-holomorphic $U(1)$ isometry of Ref.6). We show, using supergraph techniques $^{7)}$, the finiteness of the theory to leading order in $1 / \mathrm{n}$, and independently, for $\mathrm{n}=2$, the three-loop finiteness of the two-point function.

\section{SUPERSPACE FORMULATION}

Consider the following Lagrangian density:

$$
L(x)=\int d^{4} \theta\left\{e^{V} \bar{\phi}_{+} \phi_{+}+e^{-V} \bar{\phi}_{-} \phi_{-}\right\}+\int d^{2} \theta\left\{S\left(\phi_{+} \phi_{-}+i b\right)+h . c .\right\}(2.1)
$$


$\mathrm{V}$ is a real (N=1, four-dimensional) superfield, $\phi_{+}$and $\phi_{-}$are $2 \mathrm{n}$ independent chiral superfields satisfying $\overline{\mathrm{D}}_{\alpha_{+} \phi_{+}}=\overline{\mathrm{D}}_{\alpha_{-} \phi_{-}}=0$, with $\mathrm{D}_{\alpha}, \overline{\mathrm{D}}_{\dot{\alpha}}$ the usual two-component spinor covariant derivatives, and $\bar{\phi}_{+}$and $\bar{\phi}_{-}$are the complex conjugate antichiral superfields. $S$ is a Lagrange multiplier chiral superfield satisfying $\overline{\mathrm{D}}_{\alpha} \mathrm{S}=\mathrm{D}_{\alpha} \overline{\mathrm{S}}=0$ and $\mathrm{n}$ is the number of components of $\phi_{+}$and $\phi_{-}$ which can be chosen to be in a representation of $\mathrm{SU}(\mathrm{N})$ such that (2.1) is $\mathrm{SU}(\mathrm{N})$ invariant. In addition, (2.1) is invariant under the following gauge transformation with chiral parameter $\Lambda(x, \theta)$

$$
\begin{array}{ll}
\delta V=i(\Lambda-\bar{\lambda}) & \delta S=0 \\
\delta \phi_{+}=-i \Lambda \phi_{+} & \delta \bar{\phi}_{+}=i \bar{\Lambda} \bar{\phi}_{+} \\
\delta \phi_{-}=i \Lambda \phi_{-} & \delta \bar{\phi}_{-}=-i \bar{\Lambda} \bar{\phi}_{-}
\end{array}
$$

The invariance of the action under one supersymmetry is manifest, but there is also a second supersymmetry which is contained in the following transformations:

$$
\begin{array}{ll}
\delta \phi_{+}=i \bar{D}^{2}\left(e^{-V} \bar{\varepsilon} \bar{\phi}_{-}\right) & \delta V=4 i(\varepsilon \bar{S}-\overline{\varepsilon S}) \\
\delta \phi_{-}=-i \bar{D}^{2}\left(e^{V} \bar{\varepsilon} \bar{\phi}_{+}\right) & \delta S=\frac{i}{2} \bar{D}^{2}(D \varepsilon D V) \\
\delta \bar{\phi}_{+}=-i D^{2}\left(e^{-V} \varepsilon \phi_{-}\right) & \delta \bar{S}=-\frac{i}{2} D^{2}(\bar{D} \bar{\varepsilon} \overline{D V}) \\
\delta \bar{\phi}_{-}=i D^{2}\left(e^{V} \varepsilon \phi_{+}\right) &
\end{array}
$$

In order that these transformations leave the action invariant the complex superparameter $\varepsilon$ must satisfy

$$
\begin{aligned}
& D^{2} \varepsilon+\bar{D}^{2} \bar{\varepsilon}=0 \\
& \bar{D} D \varepsilon=D \bar{D} \varepsilon=0
\end{aligned}
$$

whose general solution is

$$
\varepsilon=\varepsilon_{0}+\varepsilon_{1}(x) ; \bar{D} \varepsilon_{0}=\partial \varepsilon_{0}=0, D \varepsilon_{1}(x)=0
$$

That is, $\varepsilon$ is the sum of a constant chiral superfield $\varepsilon_{0}$ and an arbitrary antichiral superfield $\varepsilon_{1}(\mathrm{x})$. The action of (2.3) restricted to $\varepsilon=\varepsilon_{1}(\mathrm{x})$ reduces to a gauge transformation when the field equations are satisfied. This 
"off-shel.l central charge" 8 ) is not relevant to our investigations and will not be discussed further. However, it can be used to remove the $\theta$ independent part of $\varepsilon_{0}$ which then takes the form

$$
\varepsilon_{0}=\theta^{\alpha} \chi_{\alpha}+i \theta^{2} f
$$

where, as a consequence of (2.4a), $f$ is real.

To make contact with the work of Ref. 5) we make the following definitions:

$$
\begin{aligned}
& \phi_{1}=\left.\phi_{+}\right|_{\theta=0} ; \phi_{2}=\left.\bar{\phi}_{-}\right|_{\theta=0} \\
& \bar{\phi}_{1}=\left.\bar{\phi}_{+}\right|_{\theta=0} ; \bar{\phi}_{2}=\left.\phi_{-}\right|_{\theta=0} \\
& V_{\mu}=\left.\bar{D}_{\mu} D V\right|_{\theta=0} ; M+i N=\left.S\right|_{\theta=0} \\
& \psi=\left.\left(D \phi_{+}+\bar{D} \bar{\phi}_{-}\right)\right|_{\theta=0}
\end{aligned}
$$

Then, performing the $\theta$ integrations, eliminating all fields not defined in (2.7) (Lagrange multipliers and auxiliary fields), we find the Lagrangian of Ref. 5), (Eq. (7)), with its constraints, (Eqs (4) and (5)). In particular, in the constraint $\vec{\phi}_{i} \vec{\tau}_{i j} \phi_{j}=\vec{b}, \vec{b}$ is found to have the specific direction $(0,2 b$, $0)$, as chosen in Ref. 6).

The form of the Lagrangian (2.1) allows us to study easily the leading order in the $1 / \mathrm{n}$ expression. The effective action $\Gamma_{0}+\Gamma_{1}$ to leading order in $1 / \mathrm{n}$ is given by the tree graphs, $\Gamma_{0}$, and the one $\phi$ loop graphs with arbitrary $S$ and $V$ external lines, $\Gamma_{1}$. The Feynman rules are standard and we refer the reader to, e.g., Ref. 7). From the form of the Lagrangian $\Gamma_{1}$ is finite if it is $S$ or $\bar{S}$ dependent. To see that $\Gamma_{1}(V)$ is finite we observe that a general graph, as in Fig. 2.1a, is finite by power counting (in two dimensions) unless all the $\mathrm{D}^{2}$ and $\overline{\mathrm{D}}^{2}$ factors at the vertices are brought together by partial integration to give the maximal number of $\mathrm{p}^{2}$ factors in the numerator, according to the formulae

$$
D^{2} \bar{D}^{2} D^{2}=-p^{2} D^{2} \quad ; \quad \bar{D}^{2} D^{2} \bar{D}^{2}=-p^{2} \bar{D}^{2}
$$


This means that effectively all but one propagator are cancelled, so that a general graph with $n$ propagators is contracted to a graph with a single propagator times a factor of $(-1)^{n-1}$. Up to a common factor of $\int \mathrm{d}^{2} \mathrm{p} / \mathrm{p}^{2}$ this is equivalent to taking all $\phi \bar{\phi}$ propagators to be -1 ; thus the infinite contribution to the effective action is described by the following reduced generating function

$$
\begin{aligned}
\exp \left(-\left.\Gamma_{1}(v)\right|_{\text {div. }}\right) & =N \int d \phi_{+} d \phi_{-} d \bar{\phi}_{+} d \bar{\phi}_{-} \exp -\left[e^{v} \bar{\phi}_{+} \phi_{+}+e^{-v} \bar{\phi}_{-} \phi_{-}\right] \\
& =\operatorname{det}^{-n}\left(e^{v}\right) \operatorname{det}^{-n}\left(e^{-v}\right)=1
\end{aligned}
$$

where $\mathrm{N}$ is an appropriately chosen normalization factor. It follows that the divergent part of $\Gamma_{1}(V)$ vanishes.

\section{UNCONSTRAINED SUPERFIELDS}

To proceed we now eliminate $S$ and $V$ from (2.1). $V$ is eliminated by its field equation

$$
V=\frac{1}{2} \ln \left(\bar{\phi}_{-} \phi_{-}\right)-\frac{1}{2} \ln \left(\bar{\phi}_{+} \phi_{+}\right)
$$

while $S$ is eliminated by resolving the constraint $\phi_{+} \phi_{-}+i b=0$. This we do by introducing $2(n-1)$ chiral superfields $u$ and $v$, as follows:

$$
\begin{aligned}
& \phi_{+}=(1+u \cdot v)^{-\frac{1}{2}}(\vec{u}, 1) \\
& \phi_{-}=(1+u \cdot v)^{-\frac{1}{2}}(\vec{v}, 1)
\end{aligned}
$$

(We have chosen $\mathrm{b}=1$ for convenience.) This not only solves the constraint but also fixes the gauge for the invariance of (2.2). In terms of $u$ and $v$ and the antichiral $\overline{\mathrm{u}}, \overline{\mathrm{v}}$, the Lagrangian now takes the form

$$
y=2 \int d^{4} \theta\left[\frac{(1+u \cdot \bar{u})(1+v \cdot \bar{v})}{(1+u \cdot v)(1+\bar{u} \cdot \bar{v})}\right]^{\frac{1}{2}} \equiv 2 \int d^{4} \theta F(u, \bar{u}, v \bar{v})
$$


Zumino has shown ${ }^{9)}$ that a Lagrangian of this form with any $F$ will describe an $\mathrm{N}=1$ ( $\mathrm{N}=2$ in two dimensions) supersymmetric $\sigma$ model defined on a Kählex manifold with Kähler potential F. The particular potential of (3.3) was found previously $^{6)}$ as the Kähler potential for the $\mathrm{N}=2$ supersymmetric model, so the above superspace form of this model is not surprising. But only for a special form of $\mathrm{F}$ can we expect to have $\mathrm{N}=2$ supersymetry as this is not guaranteed by the general form with arbitrary $F$. We can find the second supersymetry by expressing the transformations of (2.3) in terms of $u$ and $v$. But because Eqs (3.2) also fix the gauge we must simultaneously perform a compensating gauge transformation to assure that we remain in the same gauge. In fact, the transformation

$$
\delta=\delta_{\text {sup. }}\left(\varepsilon_{0}\right)+\delta_{\text {gange }}\left(\Lambda=-i \bar{D}^{2}\left[\left(\frac{\beta+\bar{\beta}^{-1}}{2}\right) \bar{\varepsilon}\right)\right)
$$

is compatible with (3.2) and this transformation acting on $u, v$ is

$$
\begin{aligned}
& \delta u=\bar{D}^{2}\left[\beta \overline{\varepsilon_{0}}(\bar{v}-u)\right] \\
& \delta v=\bar{D}^{2}\left[\bar{\beta}^{-1} \bar{\varepsilon}_{0}(v-\bar{u})\right]
\end{aligned}
$$

where $\beta$ and $\bar{\beta}$ are given by

$$
\beta=\left[\frac{(1+u \cdot \bar{u})(1+u \cdot v)}{(1+v \cdot \bar{v})(1+\bar{u} \cdot \bar{v})}\right]^{\frac{1}{2}}=\frac{(1+u \cdot \bar{u})}{(1+v \cdot \bar{v})} \bar{\beta}^{-1}
$$

The superparameter $\varepsilon_{0}$ is given in (2.6). The spinor $\chi_{\alpha}$ is the second supersymmetry parameter. To see the significance of the remaining real scalar parameter $f$ we set $x=0$ and then take the $\theta=0$ part of (3.5) to get

$$
\begin{aligned}
& \left.\delta u\right|_{\theta=0}=\left.i f \beta(\bar{v}-u)\right|_{\theta=0} \\
& \left.\delta v\right|_{\theta=0}=\left.i f \bar{\beta}^{-1}(v-\bar{u})\right|_{\theta=0}
\end{aligned}
$$


This we recognize as the non-holomorphic $U(1)$ isometry of Ref. 6). The origin of this previously rather mysterious symmetry is now explained. The commutator of the first (manifest) supersymmetry with this $U(1)$ symmetry gives the second supersymmetry; that is, $f$ is the parameter for the rotation of one supersymmetry generator into the other. From this observation we see that the additional U(1) symmetry is essential for the full $\mathrm{N}=2$ supersymmetry. All the other isometries of Ref. 6) can be directly carried over to superfields, because, being holomorphic, they respect the chirality of $u$ and $v$. In fact, the notion of a holomorphic function in component language is directly related to the notion of a chiral superfield in superspace.

\section{SUPERLOOPS}

From now on, for simplicity, we will restrict the Lagrangian (3.3) to depend only on $u, v$ with single components, i.e., $n$ (of Section 2) $=2$. Expanded in powers of $u, v$ the Lagrangian is

$$
\mathcal{L}=u \bar{u}+v \bar{v}-\frac{1}{4}\left(u^{2} \bar{u}^{2}+v^{2} \bar{v}^{2}-4 u \bar{u} v \bar{v}+2 u^{2} \bar{u} v+2 \bar{u}^{2} u \bar{v}+\right.
$$$$
\left.+2 v^{2} \bar{v} u+2 \bar{v}^{2} v \bar{u}\right)+\frac{1}{8}\left(u^{3} \bar{u}^{3}+v^{3} \bar{v}^{3}+u^{2} \bar{u}^{2} v \bar{v}+v^{2} \bar{v}^{2} u \bar{u}-5 u^{2} v^{2} \bar{u} \bar{v}-\right.
$$$$
-5 \bar{u}^{2} \bar{v}^{2} u v+3 u^{3} \bar{u} v^{2}+3 u \bar{u}^{3} \bar{v}^{2}+3 v^{3} \bar{v} u^{2}+3 v \bar{v}^{3} \bar{u}^{2}+u^{3} v \bar{u}^{2}+
$$$$
\left.+u v^{3} \bar{v}^{2}+\bar{v} \bar{u}^{3} u^{2}+\bar{u} \bar{v}^{3} u^{2}\right)+\frac{1}{16}\left(7 u^{2} \bar{u}^{2} v^{2} \bar{v}^{2}-\frac{5}{4} u^{4} \bar{u}^{4}-\frac{5}{4} v^{4} \bar{v}^{4}+\right.
$$

$$
+\cdots)+\ldots,
$$

where all purely chiral or antichiral pieces have been dropped. We will now

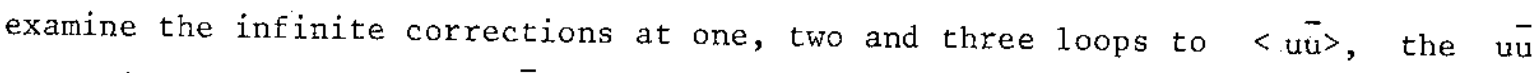
two-point function. The $\bar{v}$ two-point function is trivially the same while all other two-point functions vanish. The Feynman rules are given in Fig. 4.1.

At one loop we have the diagram shown in Fig. 4.2. There is a direct cancellation between $\mathrm{u}$ and $\mathrm{v}$ and the entire diagram vanishes. At two loops we have the diagrams shown in Fig. 4.3. In the first of these, (a), we can integrate by parts a factor of $\mathrm{D}^{2}$ or $\overline{\mathrm{D}}^{2}$ to get a factor of $-\mathrm{p}^{2}$, as in Eq. (2.8). This cancels one propagator, leading to the collapse of this diagram to one of a different form, as shown in the sequence of Fig. 4.4. On partial integration it 
may be possible that a $\mathrm{D}^{2}$ factor acts on an external line in which case the propagator cancellation does not take place. But in this case the resulting diagram is finite (in two dimensions!). Thus Fig. 4.3a can be reduced to the form of Fig. 4.3b; Fig. 4.3c vanishes by virtue of the previous one loop result. Simple combinatorics is then all that is required to show that the sum of infinite contributions vanishes.

At three loops the contributions to the u' two-point function are shown in Figs 4.5. Those of Fig. 4.5e vanish as a consequence of the previous one loop result. Upon collapsing one propagator in Fig. 4.5a it is reduced either to the form of Fig. 4.5b or 4.5c. Figure $4.5 \mathrm{~b}$ can be similarly reduced to the form of Fig. $4.5 \mathrm{~d}$ but in this case we must keep the contributions that come from partial integration of spinor derivatives onto external lines because of the subdivergence at one vertex. These one loop subdivergences cancel against other one loop subdivergences coming from collapsing the second propagator in Fig. 4.5a. This is a consequence of the one-loop finiteness of the four-point functions, as shown in Fig. 4.6. Figure 4.5c can, by partial integration of spinor derivatives, be reduced either to the form of Fig. $4.5 \mathrm{~d}$ or to that of Fig. 4.7a. But by manipulation of the integrand, as shown schematically in Fig. $4.7 \mathrm{~b}$, the latter integral can also be reduced to the form of Fig. 4.5d. As all contributions to the infinite part of the two-point function have now been reduced to a common form it remains to calculate the combinatoric factors. We find

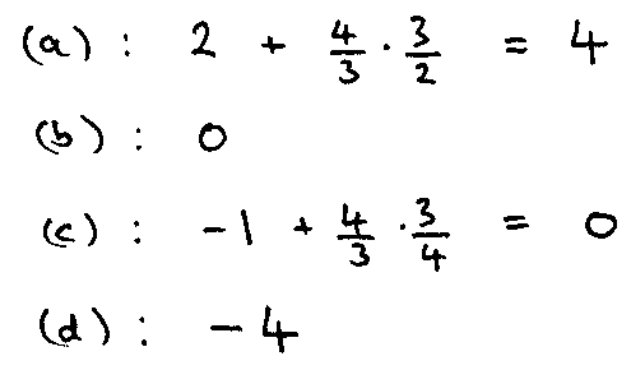

Therefore, the three-loop contribution to the two-point function is finite! Presumably the non-Iinear invariance of the theory, Eq. (3.5), can be used to relate the two-point function to the other higher point functions and thereby to deduce their finiteness to three loops.

\section{DISCUSSTON}

Our approach to the $\mathrm{N}=4$ two-dimensional non-linear supersymmetric $\sigma$ model is similar to a recent treatment of the $N=4$ four-dimensional superYang-Mills theory ${ }^{2)}$. In particular, the Lagrangian in the form of (2.1) and the 
transformation laws of (2.3) are strikingly similar. If indeed the supersymmetric $\sigma$ model is completely finite, a hope that is encouraged by our results, then a proof to all orders in perturbation theory in this formulation may be generalizable to the $N=4$ super-Yang-Mills theory. A similar formulation of the $N=8$ supergravity theory should also be possible.

\section{ACKNOWLEDGEMENTS}

One of us (P.K.T) would like to thank Prof. S. Hawking and the members of DAMTP, Cambridge for their hospitality during the course of this work. 


\section{REFERENCES}

1) E. Poggio and H. Pendleton, Phys. Lett. 72B (1977) 200;

D.R.T. Jones, Phys. Lett. 72B (1977) 199.

2) M. Grisaru, M. Roček and W. Siegel, "Zero 3-loop B-function in $N=4$ supersymmetric Yang-Mills theory", Brandeis preprint, (July, 1980).

3) P. Di Vecchia and S. Ferrara, Nucl. Phys. B130 (1977) 93;

E. Witten, Phys. Rev. D16 (1977) 2991 and Nucl. Phys. B147 (1979) 285;

A. D'Adda, P. Di Vecchia and M. Lüscher, Nuc1. Phys. B152 (1979) 125.

4) L. Alvarez-Gaumé and D.Z. Freedman, "Kähler geometry and the renormalization of supersymmetric o-mode1s", Phys. Rev. D, in press.

5) T.L. Curtright and D.Z. Freedman, Phys. Lett. 90B (1980) 71.

6) L. Alvarez-Gaumé and D.Z. Freedman, "Ricci flat Kähler manifolds and supersymmetry", Phys. Lett., in press.

7) M. Grisaru, W. Siegel and M. Roł̌ek, Nuc1. Phys. B159 (1979) 429.

8) M. Sohnius, K. Stelle and P.C. West, Proceedings of the Erice Conference on "Unification of the Fundamental Interactions" (1980).

9) B. Zumino, Phys. Lett. 87B (1979) 203.

\section{Figure captions}

Fig. 2.1 : A typical contribution to $\Gamma_{I}(\mathrm{~V})$.

Fig. 4.1 : Feynman rules for the Lagrangian (4.1). Only one vertex is shown. The others follow in similar fashion with the coupling constant as determined in the standard way from the Lagrangian (e.g., -1 for the vertex shown). External lines have no $\mathrm{D}^{2}$ or $\overline{\mathrm{D}}^{2}$ factors.

Fig. 4.2 : Vanishing of one-loop contribution to $\langle\bar{u} \bar{r}$.

Fig. 4.3 : Two-loop contributions to $\langle u \bar{u}\rangle$.

Fig. 4.4 : Collapsing a propagator.

Fig. 4.5 : Three-Ioop contributions to $\langle\bar{u} \bar{u}$.

Fig. 4.6 : One-loop finiteness of four-point functions.

Fig. 4.7 : (a) Integral coming from reduction of Figs 4.5 a and c;

(b) Relation between (a) and the integral of Fig. $4.5 \mathrm{~d}$. 
fig. (2.1)
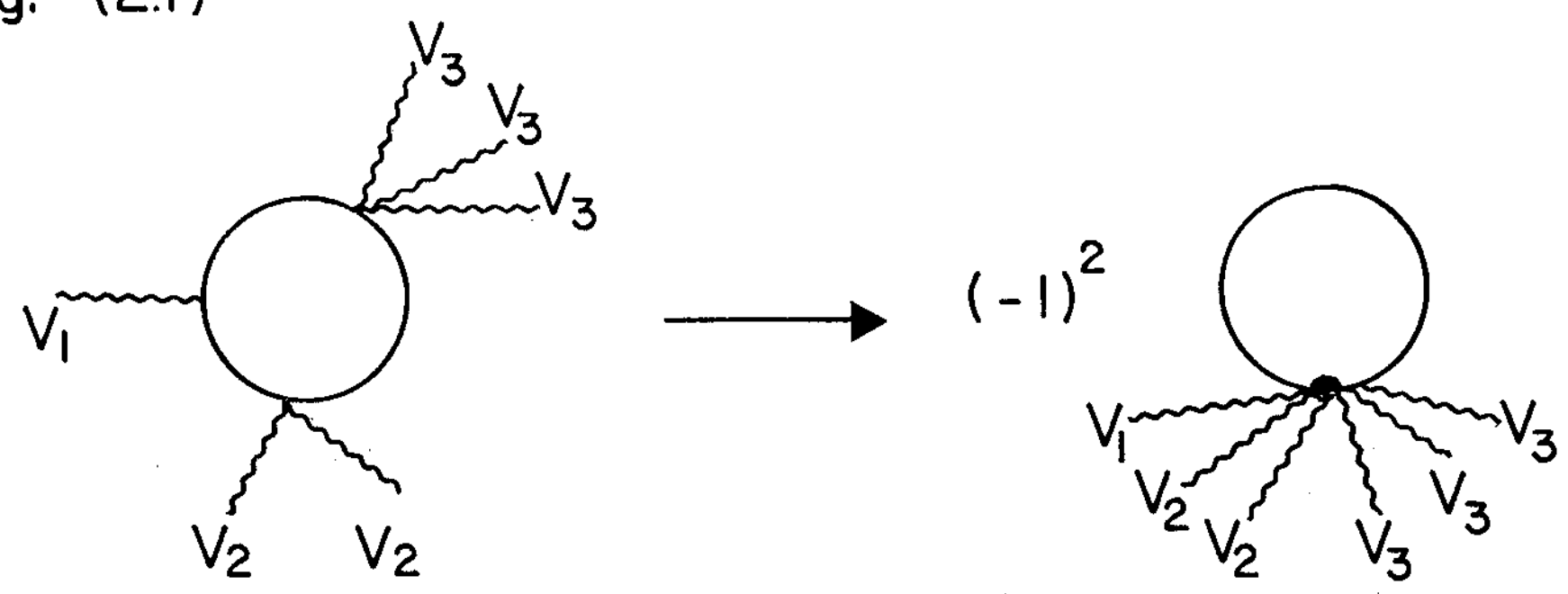

fig. (4.1)
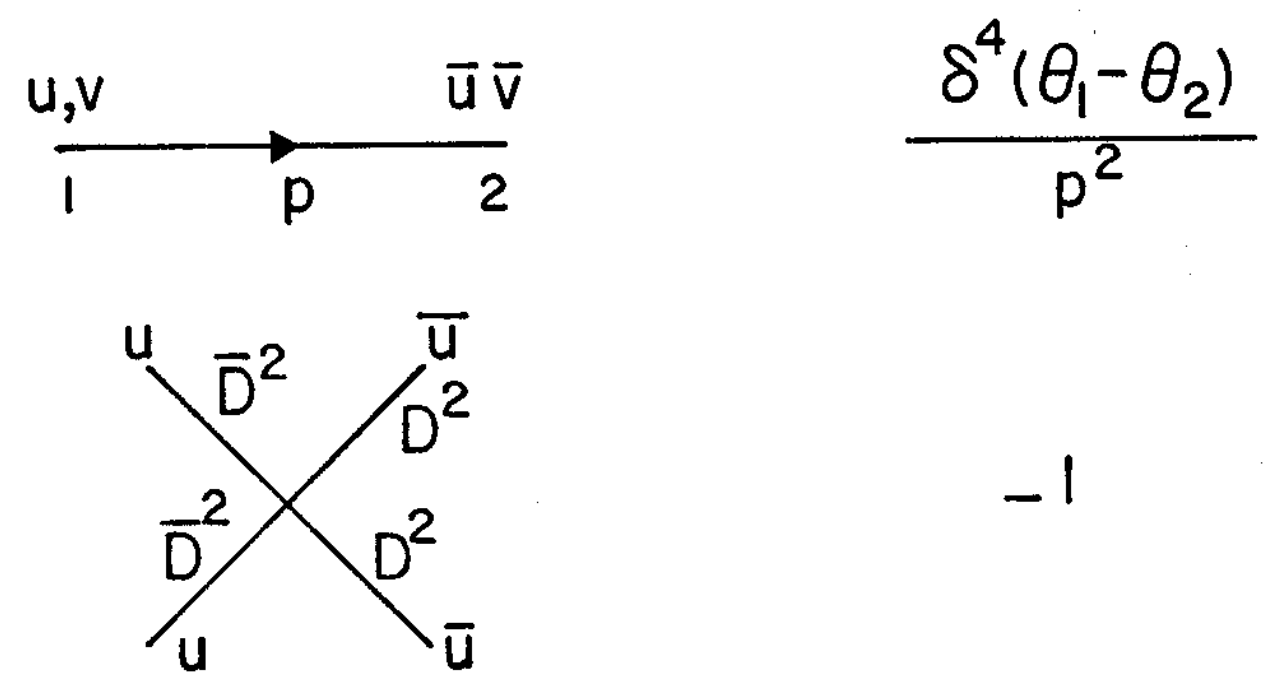

$-1$

fig. (4.2)
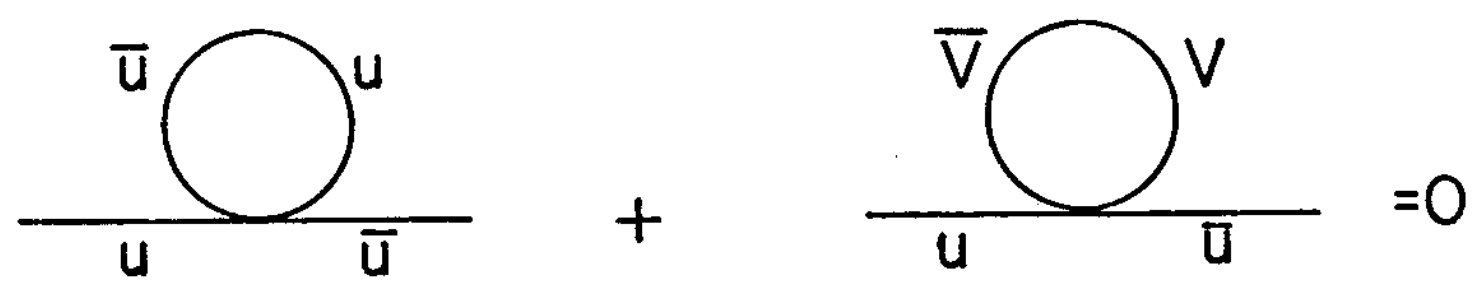
fig. (4.3)

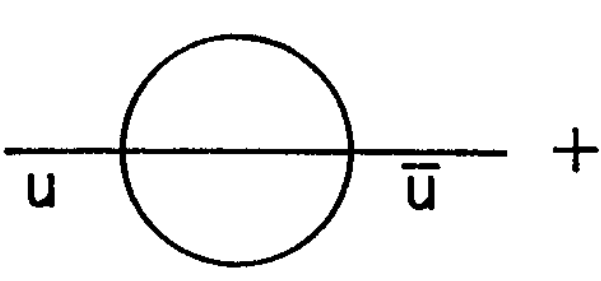

a)

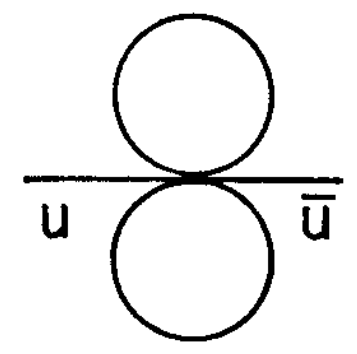

b)

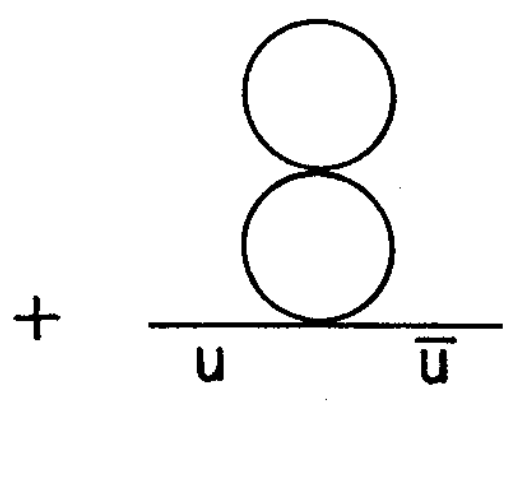

c)

fig.(4.4)
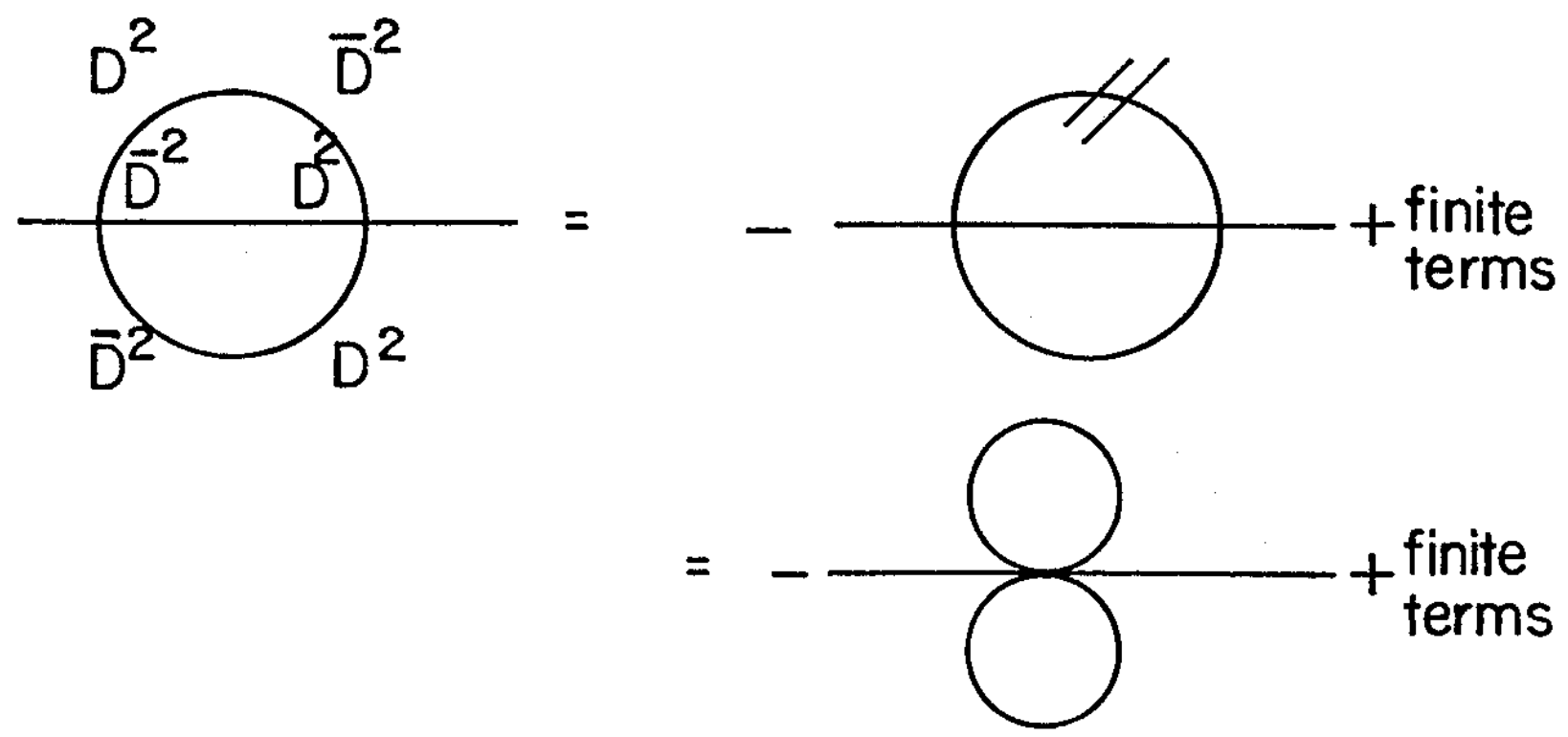
fig. (4.5)

a)

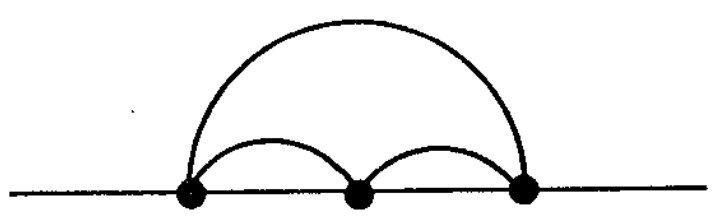

b)

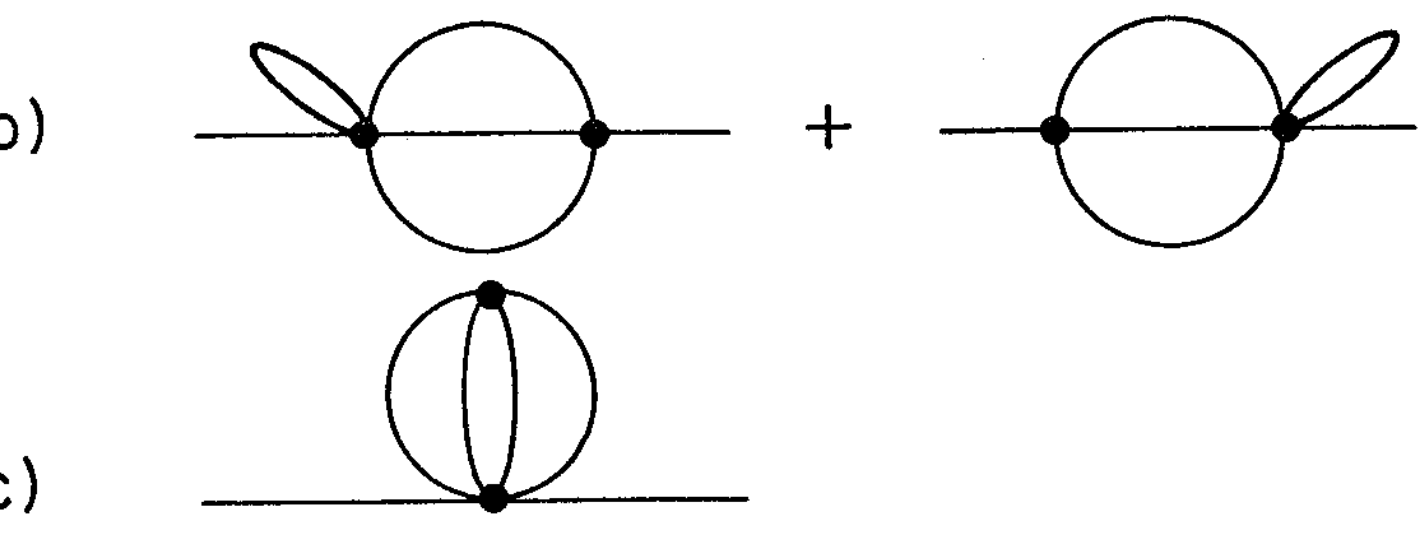

c)

d)

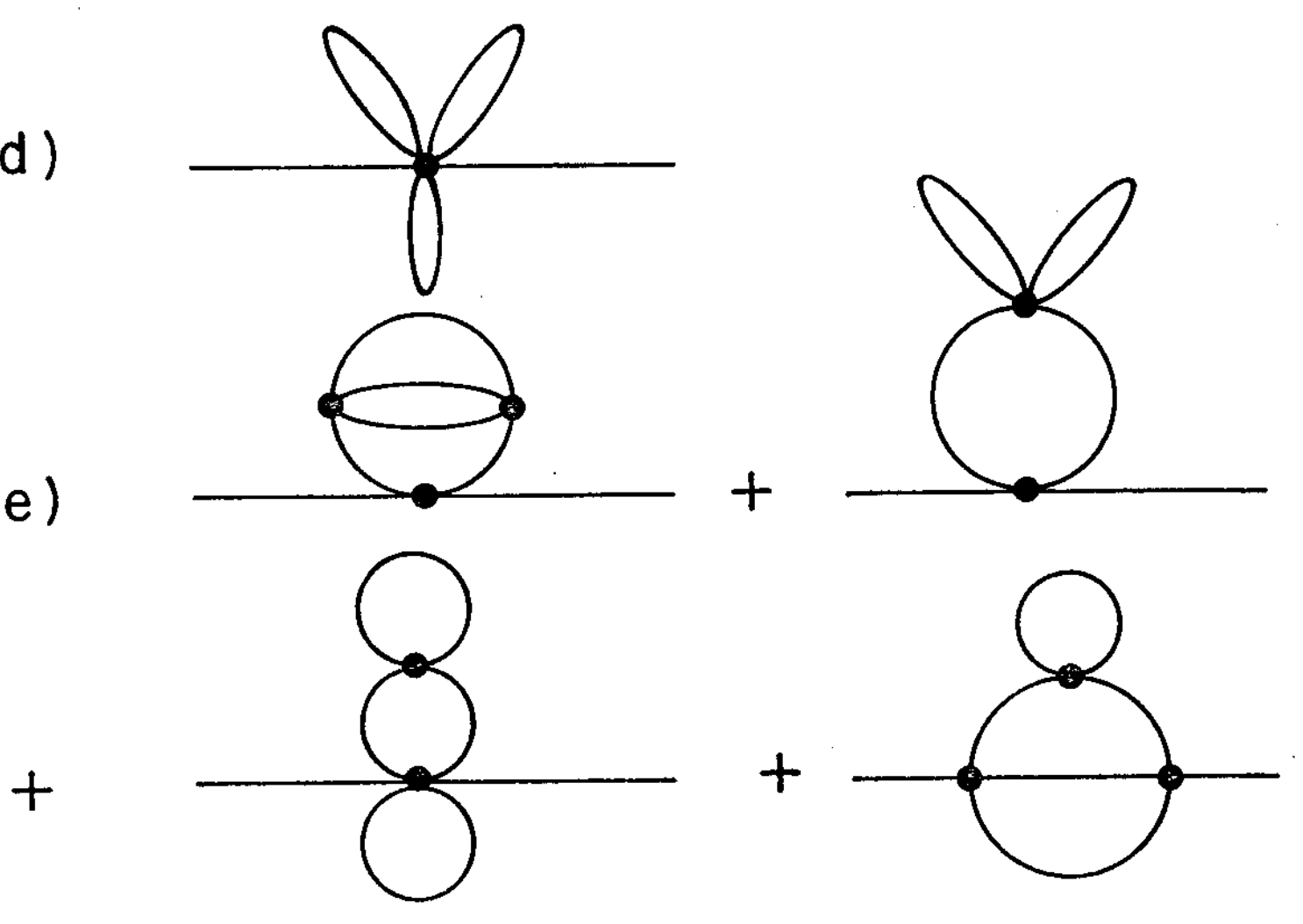

e) 
fig. (4.6)
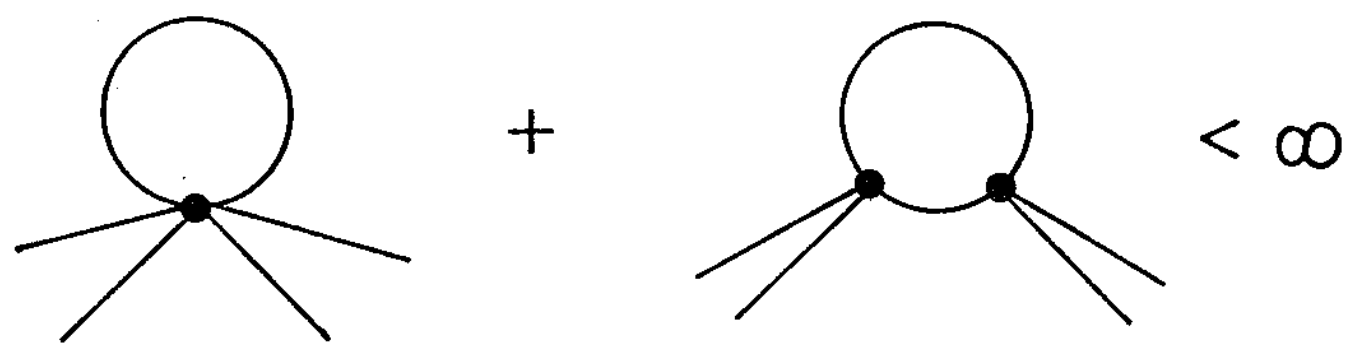

fig. (4.7)

a)

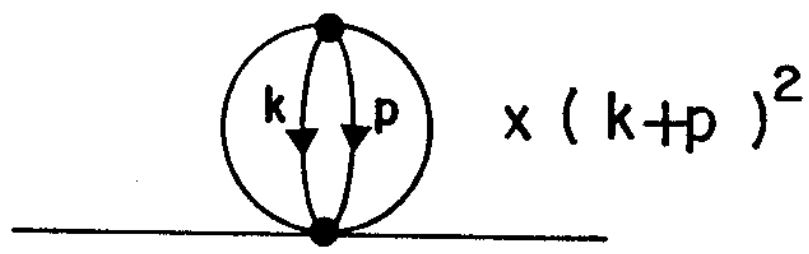

b)
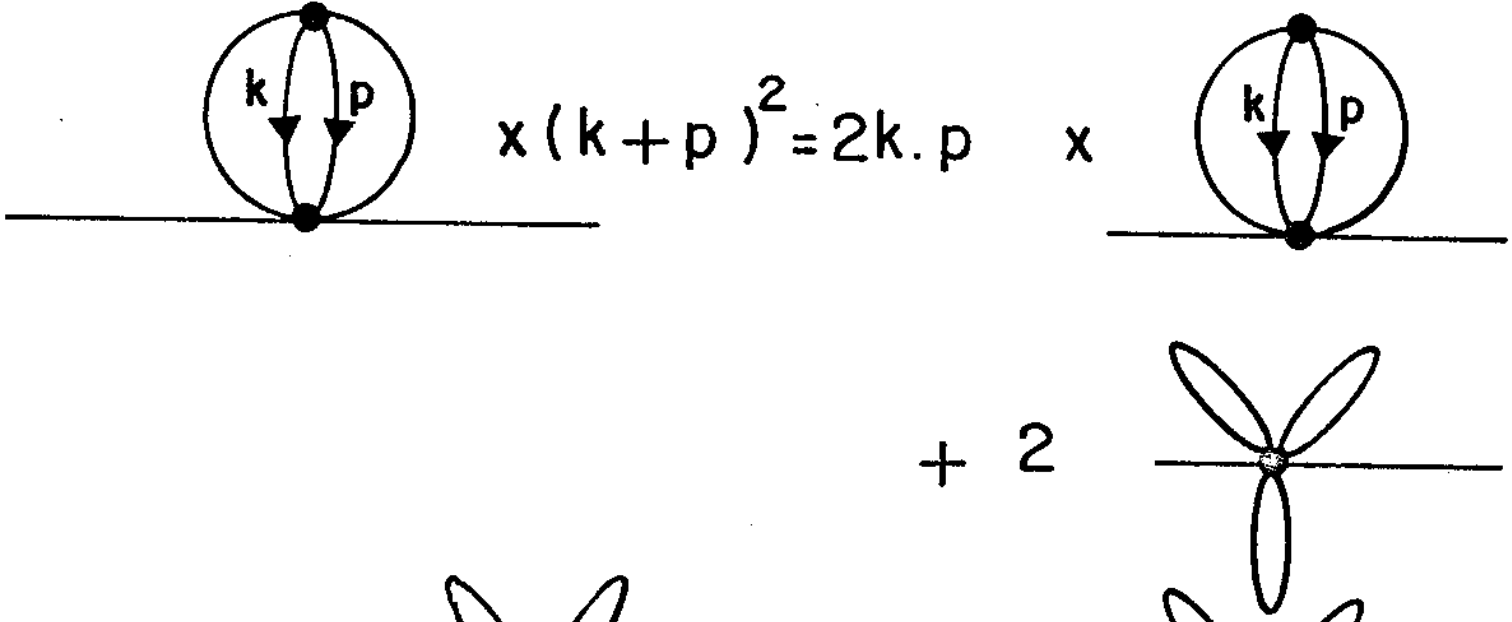

$=(-2 / 3+2)$

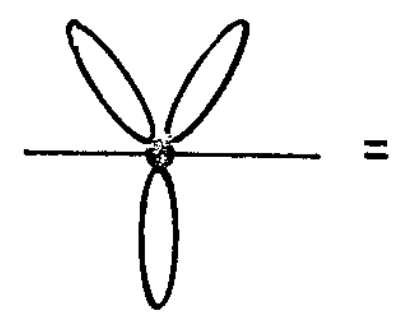

$4 / 3$<smiles>CC1(C)CCC1</smiles>

16 